\title{
Mass dependence of hadron distributions in ultrarelativistic nucleus-nucleus collisions
}

H. Sorge

Los Alamos National Laboratory, Los Alamos, NM 87545, USA and Universität Frankfurt, D-60054 Frankfurt, Germany

Z. Phys. C 67 (1995) 479

The calculated rapidity distributions of antiprotons for the reactions $\mathrm{O}(\mathrm{S})$ on $\mathrm{A}$ which are displayed in Fig. 1 are scaled by a factor of 10 (not 20 as indicated in the figure). 